\title{
Deformation theory of algebraic stacks
}

\author{
Masao Aoki
}

\begin{abstract}
We study the deformation theory of algebraic stacks. The 2-category of deformations using Ext groups of the cotangent complex is described. Then we show that an algebraic stack has a versal deformation under certain conditions.
\end{abstract}

\section{Introduction}

The aim of this paper is to generalize the deformation theory of schemes discussed in [Ill71] and [Sch68] to algebraic stacks. Deformations of algebraic stacks are defined as follows.

Definition 1.1. Let $x: \mathcal{X} \rightarrow S$ be a flat algebraic stack over a scheme $S$ and $S \hookrightarrow \widetilde{S}$ a closed immersion of schemes defined by a nilpotent ideal $I$.

A flat deformation of $\mathcal{X}$ to $\widetilde{S}$ is a pair $(\widetilde{\mathcal{X}}, F)$ where $\widetilde{\mathcal{X}}$ is a flat algebraic stack over $\widetilde{S}$ and $F: \mathcal{X} \rightarrow \widetilde{\mathcal{X}} \times_{\widetilde{S}} S$ is a 1-isomorphism of algebraic stacks.

Let $(\widetilde{\mathcal{X}}, F)$ and $\left(\widetilde{\mathcal{X}}^{\prime}, F^{\prime}\right)$ be deformations of $\mathcal{X}$ to $\widetilde{S}$. Then a morphism from $(\widetilde{\mathcal{X}}, F)$ to $\left(\widetilde{\mathcal{X}}^{\prime}, F^{\prime}\right)$ is a pair $(g, \tau)$ where $g: \widetilde{\mathcal{X}} \rightarrow \widetilde{\mathcal{X}}^{\prime}$ is a 1-morphism of algebraic stacks and $\tau$ is a 2-morphism.

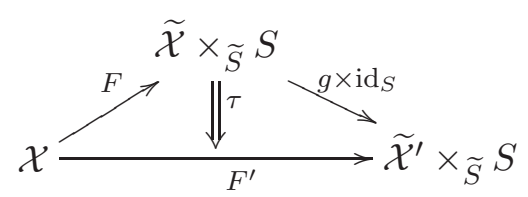

A 2-morphism from $(g, \tau)$ to $\left(g^{\prime}, \tau^{\prime}\right)$ is a 2-isomorphism $\alpha: g \Rightarrow g^{\prime}$ such that the horizontal composition $\left(\alpha \times \operatorname{id}_{i_{S}}\right) * \operatorname{id}_{F^{-1}}:\left(g \times \operatorname{id}_{S}\right) \circ F \Rightarrow\left(g^{\prime} \times \operatorname{id}_{S}\right) \circ F$ commutes with $\tau$ and $\tau^{\prime}$.

We say that $\tilde{\mathcal{X}}$ is a deformation or that $g$ is a 1-morphism of deformations as long as there is no danger of confusion.

Thus deformations of $\mathcal{X}$ to $\widetilde{S}$ form a 2-category. Our first goal is to describe this 2-category. Deformations of schemes, algebraic spaces and simplicial algebraic spaces are equivalent to those of ringed topoi of their Zariski or étale sheaves. Let $X \rightarrow S$ be a morphism of ringed topoi. Illusie [Ill71] defined the cotangent complex $L_{X / S}$ and described the category of deformations of a ringed topos $X$ using Ext groups of the cotangent complex $L_{X / S}$.

On algebraic stacks unfortunately there are only lisse-étale sheaves, and deformation theory of algebraic stacks does not coincide with that of the topoi of lisse-étale sheaves. For instance, if $X$ is

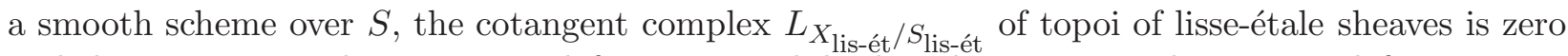
and the topos $X_{\text {lis-ét }}$ has a unique deformation, while the scheme $X$ may have more deformations.

There is, however, another cotangent complex $L_{\mathcal{X} / S}$ defined by Laumon and Moret-Bailly [LM00, $\S 17]$. In $\S 3$ we prove the following theorem. This is a generalization of the results of deformation theory of algebraic spaces.

Received 13 June 2003, accepted in final form 12 November 2003, published online 1 December 2004.

2000 Mathematics Subject Classification 14A20, $14 \mathrm{~B} 12$.

Keywords: algebraic stacks, deformation theory, cotangent complex.

The author is supported by Grant in Aid for JSPS Fellows.

This journal is (C) Foundation Compositio Mathematica 2005. 
TheOREM 1.2. Let $x: \mathcal{X} \rightarrow S$ be a flat algebraic stack over a quasiseparated scheme $S$ and $S \hookrightarrow \widetilde{S}$ a closed immersion of schemes defined by a square-zero ideal $I$. Then we have the following.

1) There exists an obstruction $o \in \operatorname{Ext}^{2}\left(L_{\mathcal{X} / S}, x^{*} I\right)$, whose vanishing is equivalent to the existence of a flat deformation of $\mathcal{X}$ to $\widetilde{S}$.

2) If $o=0$, the set of isomorphism classes of flat deformations of $\mathcal{X}$ to $\widetilde{S}$ is a torsor under $\operatorname{Ext}^{1}\left(L_{\mathcal{X} / S}, x^{*} I\right)$.

3) The group of 2-isomorphism classes of automorphisms of any flat deformation is naturally isomorphic to $\operatorname{Ext}^{0}\left(L_{\mathcal{X} / S}, x^{*} I\right)$.

4) The 2-automorphism group of an automorphism is naturally isomorphic to $\operatorname{Ext}^{-1}\left(L_{\mathcal{X} / S}, x^{*} I\right)$.

Our second goal is to show that an algebraic stack over a field has a versal deformation under certain conditions. Schlessinger [Sch68, $\S 3.7$ ] proved that, if a scheme $X$ is proper over a field $k$, it has a versal deformation. In $\S 4$ we prove the following theorem.

Theorem 1.3. If $\mathcal{X}$ is proper over a field $k$, it has a versal deformation.

We verify Schlessinger's conditions for the existence of a versal deformation.

Throughout this paper we assume that all schemes, algebraic spaces and algebraic stacks are quasiseparated. We denote the category of schemes by (Sch), that of algebraic spaces by (Alg.Spc) and that of simplicial algebraic spaces by (S.Alg.Sp). We denote the 2-category of algebraic stacks by (Alg.St). We endow the category (Sch) with the étale topology. We refer to [LM00] for the definition and properties of algebraic stacks and to [Knu71] for those of algebraic spaces. We also refer to $[$ Del74, $\S 5.1]$ for the definitions of $\left(k\right.$-) simplicial objects and functors $\mathrm{sq}_{k}, \operatorname{cosq}{ }_{k}$.

\section{Preliminaries}

First of all, let us introduce several categories related to (Alg.St).

\subsection{Groupoid space}

Definition 2.1.1. A groupoid space is a 2-simplicial space $X_{\bullet}$ such that morphisms

$$
\begin{gathered}
\left(d_{2}^{0}, d_{2}^{2}\right): X_{2} \rightarrow X_{1} \times_{d_{1}^{1} X_{0} d_{1}^{0}} X_{1}, \\
\left(d_{2}^{1}, d_{2}^{2}\right): X_{2} \rightarrow X_{1} \times_{d_{1}^{1} X_{0} d_{1}^{1}} X_{1}
\end{gathered}
$$

are isomorphisms.

Morphisms of groupoid spaces are those of 2-simplicial spaces.

Let $X_{\bullet}$ be a groupoid space. We denote the maps $d_{1}^{0}, d_{1}^{1}, s_{0}^{0}$ and $d_{2}^{1}$ by $t, s, e$ and $m$. The condition (2.1.1.2) is equivalent to prescribing a morphism $i: X_{1} \rightarrow X_{1}$ which satisfies certain equalities [SGA3, vol. 1, p. 253].

A groupoid space is determined by spaces $X_{0}, X_{1}$ and morphisms of spaces

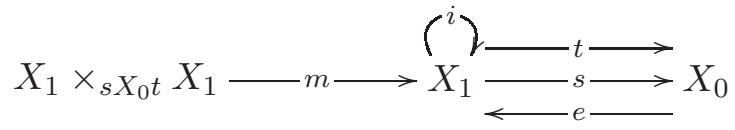

which satisfy the equalities in [LM00, Example 2.4.3].

Definition 2.1.2. An algebraic groupoid space is a groupoid space where $X_{0}$ and $X_{1}$ are algebraic spaces. Morphisms of algebraic groupoid spaces are those of groupoid spaces. We denote the category of algebraic groupoid spaces by (Alg.Gr.Sp).

An algebraic groupoid space with flat structure is an algebraic groupoid space in which the morphisms $s$ and $t$ are flat. An algebraic groupoid space with SQCS structure is an algebraic groupoid 


\section{DEFORMATION THEORY OF ALGEBRAIC STACKS}

space where the morphisms $s$ and $t$ are smooth and $(s, t): X_{1} \rightarrow X_{0} \times X_{0}$ is quasicompact and separated. We denote the full subcategory of (Alg.Gr.Sp) whose objects have flat (respectively SQCS) structures by (Alg.Gr.Sp) F (respectively (Alg.Gr.Sp) $)_{S}$.

DEFINITION 2.1.3. An étale equivalence relation (of schemes) is an algebraic groupoid space with SQCS structure $\left(X_{0}, X_{1}, s, t, e, m, i\right)$ where $X_{0}$ and $X_{1}$ are schemes, the morphisms $s$ and $t$ are étale and the morphism $(s, t): X_{1} \rightarrow X_{0} \times X_{0}$ is a monomorphism.

Remark 2.1.4. In Definitions 2.1.2 and 2.1.3, if $X_{0}$ and $X_{1}$ are algebraic spaces over a scheme $S$ and the morphisms $s, t, e, m$ and $i$ are also over $S$, we may replace the morphism $(s, t): X_{1} \rightarrow X_{0} \times X_{0}$ by the morphism $(s, t): X_{1} \rightarrow X_{0} \times_{S} X_{0}$. In fact, since the diagram

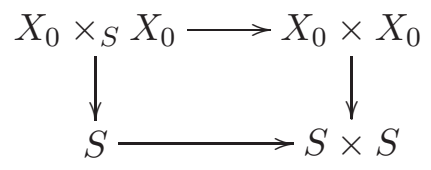

is Cartesian and the diagonal $S \rightarrow S \times S$ is quasicompact and is a monomorphism, $(s, t): X_{1} \rightarrow$ $X_{0} \times X_{0}$ is quasicompact, separated or a monomorphism if and only if $(s, t): X_{1} \rightarrow X_{0} \times_{S} X_{0}$ is.

Let $\left(X_{0}, X_{1}, s, t, e, m, i\right)$ be a groupoid space. For each scheme $U$, we define the category $\left[X_{1}, X_{0}\right]^{\prime}(U)$ as follows. The set of objects is $X_{0}(U)$. For $x, y \in X_{0}(U)$, the set of morphisms from $x$ to $y$ is $\left\{f \in X_{1}(U) \mid s(f)=x, t(f)=y\right\}$. The composition of $f \in X_{1}(U)$ and $g \in X_{1}(U)$ is given by $m(f, g)$, and the identity on $x \in X_{0}(U)$ is $e(x)$. Then $\left[X_{1}, X_{0}\right]^{\prime}(U)$ is a groupoid. In fact, the inverse of $f \in X_{1}(U)$ is given by $i(f)$.

Let $\varphi: U \rightarrow V$ be a morphism of schemes. The functor of groupoids $\left[X_{1}, X_{0}\right]^{\prime}(\varphi):\left[X_{1}, X_{0}\right]^{\prime}(V) \rightarrow$ $\left[X_{1}, X_{0}\right]^{\prime}(U)$ is given by the maps of sets $X_{0}(\varphi): X_{0}(V) \rightarrow X_{0}(U)$ and $X_{1}(\varphi): X_{1}(V) \rightarrow X_{1}(U)$.

Thus $\left[X_{1}, X_{0}\right]^{\prime}$ is a presheaf of groupoids. It is a prestack [LM00, Definition 3.1] because $X_{1}$ is a sheaf. We denote its associated stack [LM00, Lemma 3.2] by $\left[X_{1}, X_{0}\right]$. If $\left(X_{0}, X_{1}, s, t, e, m, i\right)$ is a groupoid space over a scheme $S$, its associated stack $\left[X_{1}, X_{0}\right]$ is also over $S$.

The following theorem relates algebraic stacks and groupoid spaces.

Theorem 2.1.5 (see [LM00, § 4.3]).

1) Let $\left(X_{0}, X_{1}, s, t, e, m, i\right)$ be an algebraic groupoid space with SQCS structure. Then the associated stack $\left[X_{1}, X_{0}\right]$ is an algebraic stack. Moreover, the natural map $X_{0} \rightarrow\left[X_{1}, X_{0}\right]$ gives a presentation.

2) Conversely, let $\mathcal{X}$ be an algebraic stack and let $P: X_{0} \rightarrow \mathcal{X}$ be a presentation. Then $\left(X_{0}, X_{1}, s, t, e, m, i\right)$ is a groupoid space with SQCS structure, where $X_{1}=X_{0} \times \mathcal{X} X_{0}, s$ and $t$ are the second and first projections, $e$ the diagonal, $m=p_{13}: X_{1} \times_{s X_{0} t} X_{1} \cong X_{0} \times \mathcal{X} X_{0} \times \mathcal{X} X_{0} \rightarrow$ $X_{0} \times \mathcal{X} X_{0}=X_{1}$, and $i$ exchanges factors. Moreover, $\mathcal{X} \cong\left[X_{1}, X_{0}\right]$, and the natural presentation $X_{0} \rightarrow\left[X_{1}, X_{0}\right] \cong \mathcal{X}$ is equal to $P$.

Proposition 2.1.6.

1) If $\left(X_{0}, X_{1}, s, t, e, m, i\right)$ is an étale equivalence relation of schemes, then the associated stack $\left[X_{1}, X_{0}\right]$ is isomorphic to an algebraic space.

2) If $X_{0} \rightarrow X$ is a representable étale covering, then the groupoid space $\left(X_{0}, X_{1}, s, t, e, m, i\right)$ constructed in Theorem 2.1.5, part 2 is an étale equivalence relation of schemes.

Proof. 1) By [Knu71, ch. II, Proposition 1.3] there is an algebraic space $A$ such that $X_{0} \rightarrow A$ is a representable étale cover, $X_{1} \cong X_{0} \times_{A} X_{0}$ and the morphisms $s, t, e, m, i$ are equal to those in Theorem 2.1.5, part 2. By Theorem 2.1.5, part 2, such an $A$ is isomorphic to $\left[X_{1}, X_{0}\right]$.

2) See [Knu71, ch. II, Proposition 1.3]. 


\section{AOKI}

\subsection{Deformation of algebraic spaces}

We study some properties of deformation of algebraic spaces. Let $S$ be a scheme and $S \hookrightarrow \widetilde{S}$ a closed immersion of schemes defined by a nilpotent ideal $I$.

Proposition 2.2.1. Let $x: X \rightarrow S$ be a flat algebraic space or a flat simplicial algebraic space over $S$. Suppose that $I$ is square-zero. Then the category of deformations of $X$ to $\widetilde{S}$ is a full subcategory of the category of $O_{\widetilde{S}^{-}}$extensions of $O_{X}$ by the ideal $x^{*} I$ in the category of étale $O_{X}$-modules.

Proof. [Ols02, Lemma 2.19 and $\S 3.1]$. The image of a deformation

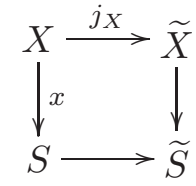

is given by an extension

$$
0 \rightarrow x^{*} I \rightarrow j_{X}^{-1} O_{\widetilde{X}} \rightarrow O_{X} \rightarrow 0
$$

LEMMA 2.2.2.

1) Let $X$ be a flat (simplicial) algebraic space over $S$. All morphisms of deformations of $X$ are isomorphisms.

2) Let $f: X \stackrel{\sim}{\rightarrow} Y$ be an isomorphism of (simplicial) algebraic spaces and $\widetilde{f}: \widetilde{X} \rightarrow \widetilde{Y}$ a deformation to $\widetilde{S}$. Then $\tilde{f}$ is also an isomorphism.

Proof. We may suppose that $I$ is square-zero. In fact, the closed immersion $S \hookrightarrow \widetilde{S}$ is a composition of closed immersions $S_{n} \hookrightarrow S_{n+1}$ defined by square-zero ideals, and any deformation is a composition of the diagrams

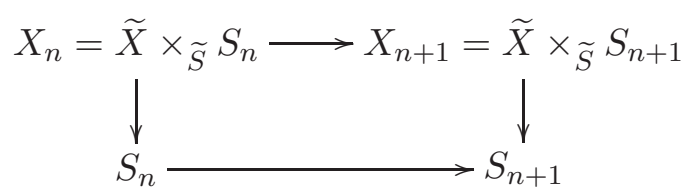

where $X_{n+1}$ is a deformation of $X_{n}$ to $S_{n+1}$.

1) Let $j_{X}: X \hookrightarrow \widetilde{X}$ and $j_{X}^{\prime}: X \hookrightarrow \widetilde{X}^{\prime}$ be deformations of $X$ to $\widetilde{S}$ and, let $\widetilde{f}: \widetilde{X} \rightarrow \widetilde{X}^{\prime}$ be a morphism of deformations. Then $\tilde{f}$ induces a morphism of extensions of $O_{X}$ by $x^{*} I$.

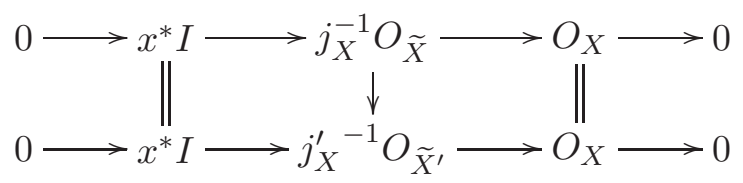

By the 5-lemma, this is an isomorphism of extensions.

2) The composition $X \stackrel{\sim}{\rightarrow} Y \hookrightarrow \widetilde{Y}$ gives a deformation of $X$ to $\widetilde{S}$. The morphism $\widetilde{f}$ is a morphism of deformations of $X$, hence an isomorphism.

Lemma 2.2.3. Let $X$ be a scheme over $S$ and $\widetilde{X}$ a deformation to $\widetilde{S}$ in the category of algebraic spaces. Then $\widetilde{X}$ is a scheme.

Proof. By [Knu71, ch. II, Proposition 6.6], there exists an open subscheme $U \hookrightarrow \widetilde{X}$ such that all scheme-like points [Knu71, ch. II, Definition 6.5] of $\widetilde{X}$ factor through $U$. Since $X$ is a subscheme of $\widetilde{X}$, it is a subscheme of $U$. Therefore $U \times_{\widetilde{S}} S \cong X$. On the other hand, $U$ is flat over $\widetilde{S}$ since $\widetilde{X}$ is. Thus $U$ is a flat deformation of $X$ to $\widetilde{S}$. Hence the open immersion $U \hookrightarrow \widetilde{X}$ is an isomorphism. 


\section{Deformation THEORY of ALGEBRAIC STACKS}

Lemma 2.2.4. Let $f: X \rightarrow Y$ be a morphism of algebraic spaces over $S$ and $\tilde{f}: \tilde{X} \rightarrow \widetilde{Y}$ a deformation to $\widetilde{S}$. Let $P$ be one of the following properties: 1) flat, 2) locally of finite presentation, 3) surjective, 4) smooth, 5) étale. Then if $f$ has the property $P$, so has $\tilde{f}$.

Proof. Since properties 1-5 are local in étale topology, we may assume that $X, Y$ and $S$ are affine schemes. Consider the diagram

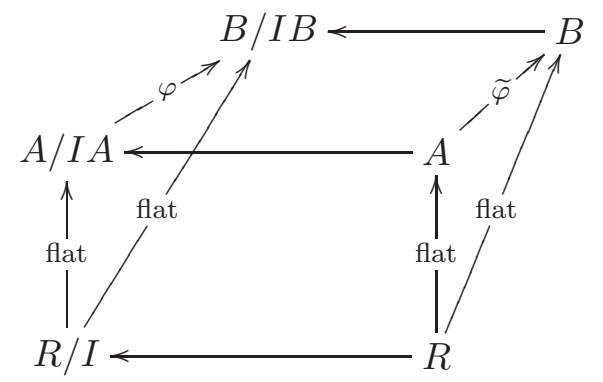

where $A, B$ and $R$ are commutative rings and $I$ is a nilpotent ideal of $R$.

To prove property 1 , we use the following proposition.

Proposition [Bou72, ch. III, $\S 5$, Theorem 1]. Let $R$ be a ring, $I$ a nilpotent ideal of $R$, and $M$ an $R$-module. Then $M$ is flat over $R$ if and only if $M / I M$ is flat over $R / I$ and the natural homomorphism

$$
I \otimes_{R} M \rightarrow I M
$$

is an isomorphism.

Returning to the proof of Lemma 2.2.4, it is enough to show that the natural homomorphism

$$
A I \otimes_{A} B \rightarrow B I
$$

is an isomorphism. Since $A$ and $B$ are flat over $R$, there is a natural isomorphism

$$
A I \otimes_{A} B \cong\left(I \otimes_{R} A\right) \otimes_{A} B \cong I \otimes_{R} B \cong B I .
$$

Properties 2 and 3 are obvious.

Properties 4 and 5 follow from 2 and [DiG61, ch. IV, Proposition 17.8.2].

Lemma 2.2.5. Let $X, Y$ and $Z$ be flat algebraic spaces over $S, f: X \rightarrow Y$ a flat morphism and $g: Z \rightarrow Y$ any morphism over $S$. If $\widetilde{f}: \widetilde{X} \rightarrow \widetilde{Y}$ and $\widetilde{g}: \widetilde{Z} \rightarrow \widetilde{Y}$ are deformations of $f$ and $g$ to $\widetilde{S}$, then the fiber product $\widetilde{X} \times_{\widetilde{Y}} \widetilde{Z}$ is a deformation of $X \times_{Y} Z$ to $\widetilde{S}$.

Proof. It is easy to check that the natural morphism

$$
\left(\widetilde{X} \times_{\widetilde{Y}} \widetilde{Z}\right) \times_{\widetilde{S}} S \rightarrow X \times_{Y} Z
$$

is an isomorphism. The morphism $\widetilde{f}$ is flat by Lemma 2.2 .4 , property 1 . Therefore, $\widetilde{X} \times_{\widetilde{Y}} \widetilde{Z}$ is flat over $\widetilde{Z}$, and hence flat over $\widetilde{S}$.

Remark 2.2.6. This lemma does not hold if $f$ is not flat, even if $X \times_{Y} Z$ is flat over $S$. For example, let $k$ be a field and let $S=\operatorname{Spec} k[x], X=Z=\operatorname{Spec} k[x, y] /(y)$ and $Y=\operatorname{Spec} k[x, y]$. Then $\widetilde{X}=\operatorname{Spec} k[\epsilon][x, y] /(y), \widetilde{Y}=\operatorname{Spec} k[\epsilon][x, y]$ and $\widetilde{Z}=\operatorname{Spec} k[\epsilon][x, y] /(\epsilon x+y)$ are deformations of $X, Y$ and $Z$ to $\widetilde{S}=\operatorname{Spec} k[\epsilon][x]$, where $k[\epsilon]$ denotes the ring of dual numbers $k[\epsilon] /\left(\epsilon^{2}\right)$. Now $X \times_{Y} Z=\operatorname{Spec} k[x, y] /(y)$ is flat over $S$. But $\widetilde{X} \times_{\widetilde{Y}} \widetilde{Z}=\operatorname{Spec} k[\epsilon][x, y] /(\epsilon x, y)$ is not flat over $\widetilde{S}$, and hence is not a deformation of $X \times_{Y} Z$.

Lemma 2.2.7. Let $X$ be a flat algebraic space over $S$ and $U \rightarrow X$ an étale covering by a scheme. If $\widetilde{X}$ is a deformation of $X$ to $\widetilde{S}$, then there exists a unique deformation $\widetilde{U}$ of $U$ to $\widetilde{S}$ such that $\widetilde{U} \rightarrow \widetilde{X}$ is an étale covering. 
Proof. By [Ols02, Theorem 1.4] and the fact that $L_{U / X}=0$, there exists a unique deformation $\widetilde{U}$ of $U$ to $X$. Such a $\widetilde{U}$ is also a deformation of $U$ to $\widetilde{S}$, and $\widetilde{U} \rightarrow \widetilde{X}$ is étale and surjective by Lemma 2.2.4, properties 3 and 5 .

\section{LEMMA 2.2.8.}

1) Let $X$ be an algebraic space over $S$ and $\widetilde{X}$ a deformation to $\widetilde{S}$. If $X$ is quasicompact, then so is $\widetilde{X}$.

2) Let $f: X \rightarrow Y$ be a morphism of algebraic spaces over $S$ and $\widetilde{f}: \widetilde{X} \rightarrow \widetilde{Y}$ a deformation to $\widetilde{S}$. If $f$ is quasicompact, then so is $\widetilde{f}$.

Proof. 1) If $X$ is a scheme, then $\widetilde{X}$ is also a scheme by Lemma 2.2.3. The schemes $X$ and $\widetilde{X}$ are homeomorphic. Therefore, if $X$ is quasicompact, then so is $\widetilde{X}$.

In general, if $X$ is a quasicompact algebraic space, then there exists an étale covering $U \rightarrow X$ such that $U$ is a quasicompact scheme. By Lemma 2.2.7, there exists an étale covering $\widetilde{U} \rightarrow \widetilde{X}$ where $\widetilde{U}$ is a deformation of $U$ to $\widetilde{S}$ and $\widetilde{U}$ is a quasicompact scheme.

2) Let $\widetilde{U} \rightarrow \widetilde{Y}$ be an étale morphism from a quasicompact scheme. Then $U=\widetilde{U} \times \widetilde{S} S \rightarrow Y$ is an étale morphism from a quasicompact scheme. Since $f$ is quasicompact, $U \times_{Y} X$ is a quasicompact algebraic space. By Lemma 2.2.5, $\widetilde{U} \times_{\widetilde{Y}} \widetilde{X}$ is a deformation of $U \times_{Y} X$, and hence is quasicompact by part 1 .

Lemma 2.2.9. Let $f: X \rightarrow Y$ be a morphism of algebraic spaces over $S$ and $\tilde{f}: \widetilde{X} \rightarrow \widetilde{Y}$ a deformation to $\widetilde{S}$. If either 1) $f$ is separated or 2) $f$ is a monomorphism, then so is $\widetilde{f}$.

Proof. 1) The morphism $\tilde{f}$ is separated if and only if the diagonal $\widetilde{\Delta}: \widetilde{X} \rightarrow \widetilde{X} \times_{\widetilde{Y}} \tilde{X}$ is a closed immersion. Let $u: \widetilde{U} \rightarrow \widetilde{X} \times_{\widetilde{Y}} \widetilde{X}$ be a morphism from a scheme. We show that the induced morphism $\widetilde{\Delta^{\prime}}: \widetilde{V}=\widetilde{U} \times_{\widetilde{X}} \times_{\widetilde{Y}} \widetilde{X} \widetilde{X} \rightarrow \widetilde{U}$ is a closed immersion of schemes.

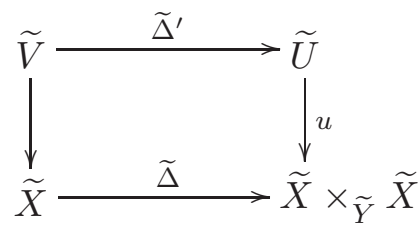

Since $\widetilde{\Delta}$ is an immersion, $\widetilde{V}$ is a scheme and $\widetilde{\Delta}^{\prime}$ is an immersion of schemes. So it suffices to show that $\widetilde{\Delta}^{\prime}$ is proper.

Let $U=\widetilde{U} \times_{\widetilde{S}} S, V=\widetilde{V} \times_{\widetilde{S}} S$ and $\Delta^{\prime}: V \rightarrow U$ be the morphism induced by $\widetilde{\Delta}^{\prime}$. Since the diagram

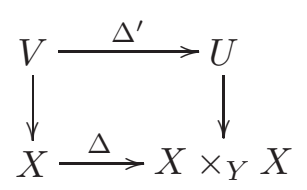

is Cartesian, $\Delta^{\prime}$ is proper. The morphisms $\Delta^{\prime}$ and $\widetilde{\Delta^{\prime}}$ induce the same morphism $\Delta_{\text {red }}^{\prime}: V_{\text {red }} \rightarrow U_{\text {red }}$. Therefore $\widetilde{\Delta}^{\prime}$ is proper, by [DiG61, ch. II, Corollary 5.4.6].

2) The morphism $\widetilde{f}$ is a monomorphism if and only if $\widetilde{\Delta}$ is an isomorphism. Let $U_{0} \rightarrow \widetilde{X} \times_{\widetilde{Y}} \widetilde{X}$ be a representable étale covering and let $U_{1}=U_{0} \times_{\widetilde{X}} \times_{\widetilde{Y}} \widetilde{X} U_{0}$. Let $\widetilde{\Delta}_{0}: V_{0}=U_{0} \times_{\widetilde{X}} \times_{\widetilde{Y}} \widetilde{X} \widetilde{X} \rightarrow U_{0}$ 
and $\widetilde{\Delta}_{1}: V_{1}=U_{1} \times_{\widetilde{X} \times_{\tilde{Y}} \widetilde{X}} \widetilde{X} \rightarrow U_{0}$ be induced morphisms.

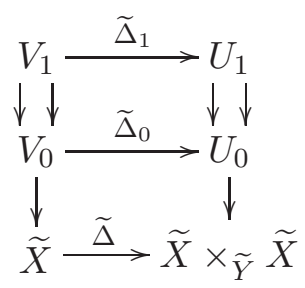

By the same argument as in part $1, V_{0}$ and $V_{1}$ are schemes. Since $\Delta: X \rightarrow X \times_{Y} X$ is an isomorphism, $\widetilde{\Delta}_{0}$ and $\widetilde{\Delta}_{1}$ induce homeomorphisms of topological spaces. Moreover, since $V_{0} \rightarrow \widetilde{X}$ is a representable étale covering and $V_{1} \cong V_{0} \times \widetilde{X} V_{0}, V_{0}$ and $V_{1}$ are flat over $\widetilde{X}$, and hence flat over $\widetilde{S}$. By [Sch68, Lemma 3.3] $\widetilde{\Delta}_{0}$ and $\widetilde{\Delta}_{1}$ induce isomorphisms of structure sheaves. Note that flatness of $U_{0}$ and $U_{1}$ is not required. We conclude that $\widetilde{\Delta}$ is an isomorphism.

\section{The 2-category of deformations}

In this section we prove Theorem 1.2. We denote the 2-category of flat deformations of $\mathcal{X}$ to $\widetilde{S}$ by $\operatorname{Defm}_{S}^{2}(\mathcal{X}, \widetilde{S})$ and its associated 1-category by $\operatorname{Defm}_{S}^{1}(\mathcal{X}, \widetilde{S})$. In other words, $\operatorname{Defm}_{S}^{1}(\mathcal{X}, \widetilde{S})$ is a category whose objects are objects of $\operatorname{Defm}_{S}^{2}(\mathcal{X}, \widetilde{S})$ and whose morphisms are 2-isomorphism classes of 1-morphisms.

Let $P: X_{0} \rightarrow \mathcal{X}$ be a presentation with $X_{0}$ an affine scheme or an infinite disjoint union of affine schemes.

We denote the algebraic groupoid space with SQCS structure $\left(X_{0}, X_{1}, s, t, e, m, i\right)$ constructed in Theorem 2.1.5, part 2 by $\mathcal{G}$ and the simplicial algebraic space $\operatorname{cosq}_{0}^{\mathcal{X}}\left(X_{0}\right)$ by $X_{\bullet}$. We denote the category of deformations of $\mathcal{G}\left(\right.$ respectively $\left.X_{\bullet}\right)$ to $\widetilde{S}$ by $\operatorname{Defm}_{S}(\mathcal{G}, \widetilde{S})\left(\operatorname{respectively} \operatorname{Defm}_{S}\left(X_{\bullet}, \widetilde{S}\right)\right.$ ). The category $\operatorname{Defm}_{S}\left(X_{\bullet}, \widetilde{S}\right)$ is described in [Ill71, ch. III, Theorem 2.1.7]. We also denote the category of deformations of $X_{0}$ to $\widetilde{\mathcal{X}}$ in the sense of [Ols02, 1.3] by $\operatorname{Defm}_{\mathcal{X}}\left(X_{0}, \widetilde{\mathcal{X}}\right)$, which is described in [Ols02, Theorem 1.4]

We first prove that the categories $\operatorname{Defm}_{S}\left(X_{\bullet}, \widetilde{S}\right)$ and $\operatorname{Defm}_{S}(\mathcal{G}, \widetilde{S})$ are categorically equivalent and that there is a functor $C: \operatorname{Defm}_{S}(\mathcal{G}, \widetilde{S}) \rightarrow \operatorname{Defm}_{S}^{1}(\mathcal{X}, \widetilde{S})$ which induces a bijection between the sets of isomorphism classes of objects. Then we show that there is an exact sequence which relates the 1-automorphism group and 2-automorphism groups of $\operatorname{Defm}_{S}^{2}(\mathcal{X}, \widetilde{S})$ to those of $\operatorname{Defm}_{S}\left(X_{\bullet}, \widetilde{S}\right)$ and $\operatorname{Defm}_{\mathcal{X}}\left(X_{0}, \widetilde{\mathcal{X}}\right)$. Finally, we describe the result using the cotangent complex of algebraic stacks [LM00, Theorem-Definition 17.3], which is independent of the choice of the presentation.

\subsection{Stabilities under deformation}

Definition 3.1.1. Let $C$ be a full (2-)subcategory of (Alg.Spc), (S.Alg.Sp) or (Alg.St). A full (2-)subcategory $C_{0}$ of $C$ is said to be stable under deformation in $C$ if, for all objects $X_{0}$ of $C_{0}$ flat over $S$, deformations of $X_{0}$ to $\widetilde{S}$ in $C$ are also in $C_{0}$.

Remark 3.1.2.

1) Let $C_{1}$ be a full subcategory of $C$ and $C_{0}$ a full subcategory of $C_{1}$. If $C_{1}$ is stable under deformation in $C$ and $C_{0}$ is stable under deformation in $C_{1}$, then $C_{0}$ is stable under deformation in $C$.

2) If $C_{0}$ is stable under deformation in $C$ and $X$ is an object of $C_{0}$ flat over $S$, then the category of flat deformations of $X$ to $\widetilde{S}$ in $C_{0}$ and that in $C$ are equivalent.

3) By Lemma 2.2.3, the category of schemes (Sch) is stable under deformation in (Alg.Spc). The category of affine schemes is stable under deformation in (Sch) [SGA7, exp. VI, $\S 4.1(\mathrm{~b})$ ].

Note that (Alg.Gr.Sp) is a subcategory of (S.Alg.Sp) by the functor $\operatorname{cosq}_{2}$. 


\section{AOKI}

Proposition 3.1.3. The category (Alg.Gr.Sp) $\mathrm{F}$ is stable under deformations in (S.Alg.Sp).

Proof. Let $\left(X_{0}, X_{1}, s, t, e, m, i\right)$ be a groupoid space, $X_{\bullet}$ its image in (S.Alg.Sp) and $\tilde{X}_{\bullet}$ a deformation of $X_{\bullet}$ in (S.Alg.Sp). The identity in $\operatorname{Hom}\left(\mathrm{sq}_{2}\left(\widetilde{X}_{\bullet}\right), \mathrm{sq}_{2}\left(\widetilde{X}_{\bullet}\right)\right)$ induces a morphism $\widetilde{f}: \widetilde{X}_{\bullet} \rightarrow$ $\operatorname{cosq}_{2}\left(\operatorname{sq}_{2}\left(\widetilde{X}_{\bullet}\right)\right)$. The right-hand side is a deformation of $\operatorname{cosq}_{2}\left(\operatorname{sq}_{2}\left(X_{\bullet}\right)\right)=X_{\bullet}$ by Lemma 2.2 .5 , and $\widetilde{f}$ is a deformation of the identity on $X_{\bullet}$, and hence an isomorphism.

The morphisms

$$
\begin{aligned}
& \left(\widetilde{d}_{2}^{0}, \widetilde{d}_{2}^{2}\right): \widetilde{X}_{2} \rightarrow \widetilde{X}_{1} \times \widetilde{d}_{1} \widetilde{X}_{0} \widetilde{d}_{1}^{0} \widetilde{X}_{1}, \\
& \left(\widetilde{d}_{2}^{1}, \widetilde{d}_{2}^{2}\right): \widetilde{X}_{2} \rightarrow \widetilde{X}_{1} \times \widetilde{d}_{1}^{1} \widetilde{X}_{0} \widetilde{d}_{1}^{1} \widetilde{X}_{1}
\end{aligned}
$$

are deformations of isomorphisms, and hence isomorphisms. Therefore $\mathrm{sq}_{2}\left(\widetilde{X_{\bullet}}\right)$ is an algebraic groupoid space with flat structure.

Corollary 3.1.4. The category (Alg.Gr.Sp) $)_{\mathrm{S}}$ is stable under deformation in (S.Alg.Sp).

Proof. If morphisms $s$ and $t$ are smooth, then so are $\widetilde{s}$ and $\widetilde{t}$ by Lemma 2.2.4, property 4 . Since $X_{0}$ is flat over $S$, the morphism $(\widetilde{s}, \widetilde{t}): \widetilde{X}_{1} \rightarrow \widetilde{X}_{0} \times \widetilde{S}_{\widetilde{X}}$ is a deformation of $(s, t): X_{1} \rightarrow X_{0} \times_{S} X_{0}$. Therefore, if $(s, t)$ is quasicompact and separated, then so is $(\widetilde{s}, \widetilde{t})$ by Lemma 2.2.8, part 2 and Lemma 2.2.9.

Corollary 3.1.5. The categories $\operatorname{Defm}_{S}\left(X_{\bullet}, \widetilde{S}\right)$ and $\operatorname{Defm}_{S}(\mathcal{G}, \widetilde{S})$ are equivalent.

COROLlary 3.1.6. The category of étale equivalence relations of schemes is stable under deformation in (S.Alg.Sp).

Proof. If $X_{0}$ and $X_{1}$ are schemes, then so are $\widetilde{X}_{0}$ and $\widetilde{X}_{1}$ by Lemma 2.2.3. If $s$ and $t$ are étale, then so are $\widetilde{s}$ and $\widetilde{t}$ by Lemma 2.2 .4 , property 5 . Finally if $(s, t): X_{1} \rightarrow X_{0} \times_{S} X_{0}$ is a monomorphism, then so is $(\widetilde{s}, \widetilde{t}): \widetilde{X}_{1} \rightarrow \widetilde{X}_{0} \times_{\widetilde{S}} \widetilde{X}_{0}$ by Lemma 2.2 .9 , part 2 .

\subsection{The functor $C$}

We define a functor $C: \operatorname{Defm}_{S}(\mathcal{G}, \widetilde{S}) \rightarrow \operatorname{Defm}_{S}^{1}(\mathcal{X}, \widetilde{S})$ as follows:

Let $\widetilde{\mathcal{G}}=\left(\widetilde{X}_{0}, \widetilde{X}_{1}, \widetilde{s}, \widetilde{t}, \widetilde{e}, \widetilde{m}, \widetilde{i}\right)$ be an object of $\operatorname{Defm}_{S}(\mathcal{G}, \widetilde{S})$. Then the associated stack $\widetilde{\mathcal{X}}=$ $\left[\widetilde{X}_{1}, \widetilde{X}_{0}\right]$ is an algebraic stack over $\widetilde{S}$. The algebraic stack $\widetilde{\mathcal{X}}$ is flat over $\widetilde{S}$ since $\widetilde{X}_{0}$ is. The morphism of groupoid spaces $\mathcal{G} \rightarrow \widetilde{\mathcal{G}}$ induces a morphism of prestacks $\left[X_{1}, X_{0}\right]^{\prime} \rightarrow\left[\widetilde{X}_{1}, \widetilde{X}_{0}\right]^{\prime}$, whence a morphism is induced between their associated stacks $\mathcal{X}=\left[X_{1}, X_{0}\right] \rightarrow\left[\widetilde{X}_{1}, \widetilde{X}_{0}\right]=\widetilde{\mathcal{X}}$. By [Gir71, ch. II, Corollary 2.2.7], taking associated stacks is commutative with base change. Therefore there is an isomorphism

$$
\widetilde{\mathcal{X}} \times_{\widetilde{S}} S=\left[\widetilde{X}_{1}, \widetilde{X}_{0}\right] \times_{\widetilde{S}} S \cong\left[\widetilde{X}_{1} \times_{\widetilde{S}} S, \widetilde{X}_{0} \times_{\widetilde{S}} S\right] \cong\left[X_{1}, X_{0}\right] \cong \mathcal{X} .
$$

Thus $\widetilde{\mathcal{X}}$ is a flat deformation of $\mathcal{X}$ to $\widetilde{S}$. This is $C(\mathcal{G})$.

Let $\left(\widetilde{X}_{0}, \widetilde{X}_{1}, \widetilde{s}, \widetilde{t}, \widetilde{e}, \widetilde{m}, \widetilde{i}\right)$ and $\left(\widetilde{X}_{0}^{\prime}, \widetilde{X}_{1}^{\prime}, \widetilde{s}^{\prime}, \widetilde{t}^{\prime}, \widetilde{e}^{\prime}, \widetilde{m}^{\prime}, \widetilde{i}^{\prime}\right)$ be objects of $\operatorname{Defm}_{S}(\mathcal{G}, \widetilde{S})$ and let $f$ be a morphism between them. Then $f$ induces a morphism $\left[\widetilde{X}_{1}, \widetilde{X}_{0}\right]^{\prime} \rightarrow\left[\widetilde{X}_{1}^{\prime}, \widetilde{X}_{0}^{\prime}\right]^{\prime}$ of prestacks and the morphism $g:\left[\widetilde{X}_{1}, \widetilde{X}_{0}\right] \rightarrow\left[\widetilde{X}_{1}^{\prime}, \widetilde{X}_{0}^{\prime}\right]$ of the associated stacks. By [Gir71, ch. II Corollary 2.2.7], the restriction of $g$ on $\mathcal{X}$ is 2-isomorphic to the morphism induced by the restriction of $f$ on $\mathcal{G}$, which is the identity. Thus $g$ gives a 2-isomorphism class of 1-morphisms of deformations of $\mathcal{X}$ to $\widetilde{S}$, which is $C(f)$.

Lemma 3.2.1. Let $\widetilde{\mathcal{X}}$ be a deformation of $\mathcal{X}$ to $S$. If the diagram

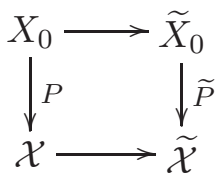

is a flat deformation of $X_{0}$ to $\widetilde{\mathcal{X}}$ in the sense of $[\mathrm{Ols} 02,1.3]$, then $\widetilde{P}$ is smooth and surjective. 


\section{DeFormation THEORY OF ALGEBRAIC STACKS}

Proof. Since $\widetilde{P}$ is a morphism from a scheme to an algebraic stack, it is representable. Hence it is enough to show that, for any scheme $\widetilde{U}$ and morphism $\widetilde{U} \rightarrow \widetilde{\mathcal{X}}$, the morphism $\widetilde{P}^{\prime}: \widetilde{X}_{0} \times \widetilde{\mathcal{U}} \rightarrow \widetilde{U}$ is smooth and surjective.

Let $U=\widetilde{U} \times \widetilde{S}_{\widetilde{S}} S$. Then $X_{0} \times \mathcal{X} U$ is a flat algebraic space over a scheme $U$ and $\widetilde{X}_{0} \times \widetilde{\mathcal{U}}$ is a deformation to $\widetilde{U}$. The morphism $P^{\prime}: X_{0} \times \mathcal{X} U \rightarrow U$ is smooth and surjective, since $P$ is. The morphism $\widetilde{P}^{\prime}$ is a deformation of $P^{\prime}$ to $\widetilde{U}$, and hence is smooth and surjective by Lemma 2.2.4 properties 4 and 3 .

Corollary 3.2.2. If $P$ is étale, then so is $\widetilde{P}$.

Lemma 3.2.3. Let $\widetilde{\mathcal{X}}$ be a flat deformation of $\mathcal{X}$ to $\widetilde{S}$ and $\widetilde{X}_{0}$ a flat deformation of $X_{0}$ to $\widetilde{\mathcal{X}}$. Let $\widetilde{\mathcal{G}}=$ $\left(\widetilde{X}_{0}, \widetilde{X}_{1}, \widetilde{s}, \widetilde{t}, \widetilde{e}, \widetilde{m}, \widetilde{i}\right)$ be a groupoid space obtained from $\widetilde{\mathcal{X}}$ and $\widetilde{P}: \widetilde{X}_{0} \rightarrow \widetilde{\mathcal{X}}$ as in Theorem 2.1.5, part 2. Then

1) $\widetilde{\mathcal{G}}$ is a flat deformation of $\mathcal{G}$ to $\widetilde{S}$ in (Alg.Gr.Sp) $)_{\mathrm{S}}$ and $C(\widetilde{\mathcal{G}})=\widetilde{\mathcal{X}}$,

2) If $\widetilde{\mathcal{G}}^{\prime}=\left(\widetilde{X}_{0}, \widetilde{X}_{1}^{\prime}, \widetilde{s}^{\prime}, \widetilde{t}^{\prime}, \widetilde{e}^{\prime}, \widetilde{m}^{\prime}, \widetilde{i}^{\prime}\right)$ is a flat deformation of $\mathcal{G}$ to $\widetilde{S}$ in (Alg.Gr.Sp) $)_{\mathrm{S}}$ such that $C\left(\widetilde{\mathcal{G}}^{\prime}\right)=\widetilde{\mathcal{X}}$ and the natural presentation $\widetilde{X}_{0} \rightarrow \widetilde{\mathcal{X}}$ is 2-isomorphic to $\widetilde{P}$, then $\widetilde{\mathcal{G}}^{\prime}$ and $\widetilde{\mathcal{G}}$ are isomorphic.

Proof. 1) Since $\widetilde{\mathcal{X}}$ is flat over $\widetilde{S}$ and the presentation $\widetilde{X}_{0} \rightarrow \widetilde{\mathcal{X}}$ is smooth, $\widetilde{X_{0}}$ is flat over $\widetilde{S}$. The isomorphisms $X_{0} \cong \widetilde{X}_{0} \times_{\widetilde{\mathcal{X}}} \mathcal{X}$ and $\mathcal{X} \cong \widetilde{\mathcal{X}} \times_{\widetilde{S}} S$ induce an isomorphism $X_{0} \cong \widetilde{X}_{0} \times_{\widetilde{S}} S$. Therefore $\widetilde{X}_{0}$ is a deformation of $X_{0}$ to $\widetilde{S}$. Moreover, since $\widetilde{X}_{0}$ is flat over $\widetilde{S}$ and $\widetilde{P}$ is smooth, $\widetilde{X}_{1}$ is flat over $\widetilde{S}$, and the restriction of $\widetilde{X}_{1}$ on $S$ is

$$
\widetilde{X}_{1} \times_{\widetilde{S}} S=\left(\widetilde{X}_{0} \times \widetilde{\mathcal{X}}_{0}\right) \times_{\widetilde{S}} S \cong X_{0} \times{ }_{\mathcal{X}} X_{0}=X_{1} .
$$

Therefore $\widetilde{X}_{1}$ is a deformation of $X_{1}$ to $\widetilde{S}$. Since the morphism $\widetilde{s}: \widetilde{X}_{0} \times \widetilde{\mathcal{X}}_{0} \rightarrow \widetilde{X}_{0}$ is the second projection, its restriction on $X_{0} \times \mathcal{X} X_{0}$ is also the second projection, which is $s$. Similarly the restrictions of $\widetilde{t}, \widetilde{e}, \widetilde{m}$ and $\widetilde{i}$ are $t, e, m$ and $i$. Therefore $\widetilde{\mathcal{G}} \times \widetilde{S} S$ is isomorphic to $\mathcal{G}$. Thus $\widetilde{\mathcal{G}}$ is a flat deformation of $\mathcal{G}$ to $\widetilde{S}$ in (Alg.Gr.Sp). The facts that $\widetilde{\mathcal{G}}$ has SQCS structure and $C(\widetilde{\mathcal{G}})=\widetilde{\mathcal{X}}$ follow from Theorem 2.1.5, part 2 .

2) The morphisms $\widetilde{s}^{\prime}, \widetilde{t}^{\prime} \in \widetilde{X}_{0}\left(\widetilde{X}_{1}^{\prime}\right)$ and the natural 2-isomorphism $\widetilde{P} \circ \widetilde{s}^{\prime} \Rightarrow \widetilde{P} \circ \widetilde{t}^{\prime}$ induce a morphism of algebraic spaces

$$
f: \tilde{X}_{1}^{\prime} \rightarrow \widetilde{X}_{0} \times \widetilde{\mathcal{X}}_{0}=\widetilde{X}_{1} .
$$

The restriction of $f$ on $X_{1}$ is induced by $t, s \in X_{0}\left(X_{1}\right)$ and the natural isomorphism $P \circ s \Rightarrow P \circ t$; hence the identity. It is easy to check the following equalities:

$$
\widetilde{s} f=\widetilde{s}^{\prime}, \quad \widetilde{t} f=\widetilde{t}^{\prime}, \quad \widetilde{e}=f \widetilde{e}^{\prime}, \quad \widetilde{m}(f, f)=f \widetilde{m}^{\prime}, \quad \widetilde{i} f=\widetilde{f i^{\prime}} .
$$

Therefore $\left(\operatorname{id}_{X_{0}}, f\right): \widetilde{\mathcal{G}}^{\prime} \rightarrow \widetilde{\mathcal{G}}$ is a morphism of groupoid spaces. Since its restriction on $\mathcal{G}$ is the identity, it is an isomorphism.

From now on, we suppose that $I$ is square-zero. The following Propositions 3.2.5, 3.2.7, 3.2.8 and 3.3.1 also hold when $I$ is a nilpotent ideal, since any closed immersion $S \hookrightarrow \widetilde{S}$ defined by a nilpotent ideal can be factored into a composition of closed immersions $S_{n} \hookrightarrow S_{n+1}$ defined by squarezero ideals $I_{n}$. Moreover, if $X_{0}$ is an affine scheme or a disjoint union of affine schemes, then so is $\widetilde{X}_{0}$.

Lemma 3.2.4. $\operatorname{Ext}^{i}\left(L_{X_{0} / \mathcal{X}}, J\right)=0$ for $i>0$ and $J$ a quasicoherent sheaf on $X_{0}$.

Proof. Since $P: X_{0} \rightarrow \mathcal{X}$ is smooth and representable, by [LM00, Lemma 17.5.8] its cotangent complex $L_{X_{0} / \mathcal{X}}$ is quasiisomorphic to an $O_{X_{0}}$-module $\Omega_{X_{0} / \mathcal{X}}$, which is locally free. Therefore

$$
\operatorname{Ext}^{i}\left(L_{X_{0} / \mathcal{X}}, J\right) \cong \operatorname{Ext}^{i}\left(\Omega_{X_{0} / \mathcal{X}}, J\right) \cong \mathrm{H}^{i}\left(X_{0}, \mathcal{H} \text { om }\left(\Omega_{X_{0} / \mathcal{X}}, O_{X_{0}}\right) \otimes J\right) .
$$


If $X_{0}$ is affine, then the right-hand side is zero for $i>0$. Otherwise, let $X_{0}=\bigsqcup_{j} U_{j}$ where each $U_{j}$ is affine. Then

$$
\mathrm{H}^{i}\left(X_{0}, \mathcal{H o m}\left(\Omega_{X_{0} / \mathcal{X}}, O_{X_{0}}\right) \otimes J\right) \cong \bigoplus_{j} \mathrm{H}^{i}\left(U_{j},\left.\mathcal{H o m}\left(\Omega_{U_{j} / \mathcal{X}}, O_{U_{j}}\right) \otimes J\right|_{U_{j}}\right)=0 .
$$

Proposition 3.2.5. The functor $C$ induces a bijection of the sets of isomorphism classes of objects.

Proof. It is enough to show that, if $\widetilde{\mathcal{X}}$ is a flat deformation of $\mathcal{X}$ to $\widetilde{S}$, then there exists a unique (up to an isomorphism) algebraic groupoid space with SQCS structure $\widetilde{\mathcal{G}}$ such that $C(\widetilde{\mathcal{G}})=\widetilde{\mathcal{X}}$. Further, by Lemma 3.2.3, it is enough to show that there exists a unique (up to an isomorphism) deformation of $X_{0}$ to $\widetilde{\mathcal{X}}$.

By [Ols02, Theorem 1.4], there exists an obstruction $o \in \operatorname{Ext}^{2}\left(L_{X_{0} / \mathcal{X}}, P^{*} x^{*} I\right)$ whose vanishing is equivalent to the existence of such a deformation, and if $o=0$, the set of isomorphism classes of deformations is a torsor under $\operatorname{Ext}^{1}\left(L_{X_{0} / \mathcal{X}}, P^{*} x^{*} I\right)$. Both of these groups are zero by Lemma 3.2.4.

\section{Corollary 3.2.6.}

1) There exists an obstruction $o \in \operatorname{Ext}^{2}\left(L_{X_{\bullet} / S_{\bullet}}, P_{\bullet}^{*} x^{*} I\right)$ whose vanishing is equivalent to the existence of a flat deformation of $\mathcal{X}$ to $\widetilde{S}$.

2) If $o=0$, then the set of isomorphism classes of flat deformations of $\mathcal{X}$ to $\widetilde{S}$ is a torsor under $\operatorname{Ext}^{1}\left(L_{X_{\bullet} / S_{\bullet}}, P_{\bullet}^{*} x^{*} I\right)$.

Proposition 3.2.7. The 2-category of Deligne-Mumford stacks is stable under deformation in (Alg.St).

Proof. Suppose that the presentation $P: X_{0} \rightarrow \mathcal{X}$ is étale. Let $\mathcal{X}$ be a deformation of $\mathcal{X}$ to $\widetilde{S}$ in (Alg.St). By Proposition 3.2.5, there exists a morphism $\widetilde{P}: \widetilde{X}_{0} \rightarrow \widetilde{\mathcal{X}}$ which gives a deformation of $X_{0}$ to $\mathcal{X}$. By Corollary 3.2.2, $\widetilde{P}$ is étale and surjective, i.e. an étale presentation.

Proposition 3.2.8. The category (Alg.Spc) is stable under deformation in (Alg.St).

Proof. Let $X$ be an algebraic space and $P: X_{0} \rightarrow X$ the representable étale covering. Then the groupoid space $\mathcal{G}$ is an étale equivalence relation by Proposition 2.1.6, part 2. Let $\widetilde{\mathcal{G}}$ be a deformation of $\mathcal{G}$ to $\widetilde{S}$. By Corollary 3.1.6, $\widetilde{\mathcal{G}}$ is an étale equivalence relation over $\widetilde{S}$. By Proposition 3.2.5, every deformation of $X$ in (Alg.St) is 1-isomorphic to an algebraic stack of the form $C(\widetilde{\mathcal{G}})$, which is an algebraic space by Proposition 2.1.6, part 1.

\subsection{The 1-automorphism groups and the 2-automorphism groups}

Proposition 3.3.1. The category $\operatorname{Defm}_{S}^{1}(\mathcal{X}, \widetilde{S})$ is a groupoid. In the other words, every 1-morphism of deformations of $\mathcal{X}$ is a 1-isomorphism.

Proof. Let $\widetilde{\mathcal{X}}$ and $\widetilde{\mathcal{X}}^{\prime}$ be deformations of $\mathcal{X}$ to $S$ and let $g: \widetilde{\mathcal{X}} \rightarrow \widetilde{\mathcal{X}}^{\prime}$ be a morphism of deformations. Let $\widetilde{P}^{\prime}: \widetilde{X}_{0}^{\prime} \rightarrow \widetilde{\mathcal{X}}^{\prime}$ be a presentation of $\widetilde{\mathcal{X}}^{\prime}$ and let $X_{0}=\widetilde{X}_{0}^{\prime} \times \widetilde{\mathcal{X}}^{\prime} \mathcal{X}$ and $\widetilde{X}_{0}=\widetilde{X}_{0}^{\prime} \times \widetilde{\mathcal{X}}^{\prime} \widetilde{\mathcal{X}}$. Then $X_{0}$ is an algebraic space, and $\widetilde{X}_{0}$ and $\widetilde{X}_{0}^{\prime}$ are deformations of $X_{0}$ to $\widetilde{S}$. By Proposition 3.2.8, $\widetilde{X}_{0}$ is an algebraic space. Moreover, $P: X_{0} \rightarrow \mathcal{X}$ and $\widetilde{P}: \widetilde{X}_{0} \rightarrow \widetilde{\mathcal{X}}$ are smooth and surjective. Therefore $P$ and $\widetilde{P}$ are presentations. 
Let $\left.X_{\bullet}=\operatorname{cosq}_{0}^{\mathcal{X}}\left(X_{0}\right), \tilde{X}_{\bullet}=\operatorname{cosq}_{0} \tilde{\mathcal{X}}^{(} \tilde{X}_{0}\right)$ and $\tilde{X}_{\bullet}^{\prime}=\operatorname{cosq}_{0}^{\tilde{\mathcal{X}}^{\prime}}\left(\tilde{X}_{0}^{\prime}\right)$.

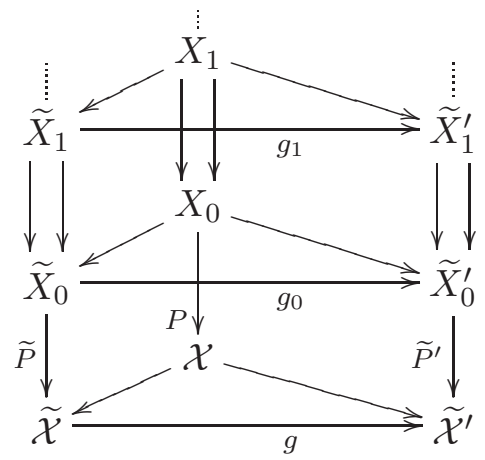

Then $\tilde{X}_{\bullet}$ and $\widetilde{X}_{\bullet}^{\prime}$ are deformations of $X_{\bullet}$ to $\widetilde{S}$ and $g$ induces a morphism of deformations $g_{\bullet}$ : $\widetilde{X}_{\bullet} \rightarrow \widetilde{X}_{\bullet}^{\prime}$. Since the category $\operatorname{Defm}_{S}\left(X_{\bullet}, \widetilde{S}\right)$ is a groupoid, $g_{\bullet}$ is an isomorphism. Therefore $g$ is an isomorphism.

Proposition 3.3.2. There is the following exact sequence

$$
\begin{aligned}
0 \rightarrow & 2-\operatorname{Aut}_{\operatorname{Defm}_{S}^{2}(\mathcal{X}, \widetilde{S})}\left(\operatorname{id}_{\widetilde{\mathcal{X}}}\right) \rightarrow \operatorname{Aut}_{\operatorname{Defm}_{\mathcal{X}}\left(X_{0}, \widetilde{\mathcal{X}}\right)}\left(\widetilde{X}_{0}\right) \\
& \stackrel{A}{\longrightarrow} \operatorname{Aut}_{\operatorname{Defm}_{S}(\mathcal{G}, \widetilde{S})}(\widetilde{\mathcal{G}}) \stackrel{C_{\widetilde{\mathcal{G}}}}{\longrightarrow} \operatorname{Aut}_{\operatorname{Defm}_{S}^{1}(\mathcal{X}, \widetilde{S})}(\widetilde{\mathcal{X}}) \rightarrow 0 .
\end{aligned}
$$

Proof. Note that a morphism $f: \widetilde{X}_{1} \rightarrow \widetilde{X}_{1}$ is given by $f_{1}, f_{2} \in \widetilde{X}_{0}\left(\widetilde{X}_{1}\right)$ and a 2-isomorphism $\widetilde{P} \circ f_{1} \Rightarrow \widetilde{P} \circ f_{2}$. Let $\epsilon: \widetilde{P} \circ \widetilde{t} \Rightarrow \widetilde{P} \circ \widetilde{s}$ be the 2-isomorphism that gives $\operatorname{id}_{\widetilde{X}_{1}}: \widetilde{X}_{1} \rightarrow \widetilde{X}_{1}$.

First we see that the homomorphism $C_{\widetilde{\mathcal{G}}}$ induced by the functor $C$ is surjective. Let $g: \widetilde{\mathcal{X}} \rightarrow \widetilde{\mathcal{X}}$ be an automorphism in $\operatorname{Defm}_{S}^{1}(\mathcal{X}, \widetilde{S})$. Since $g^{-1} \circ j$ and $j$ are 2-isomorphic, the diagrams
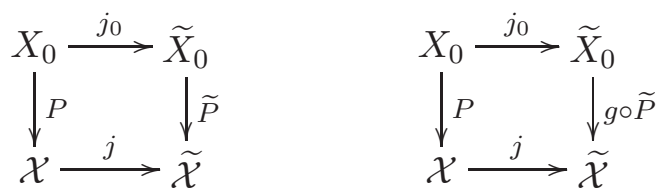

give flat deformations of $X_{0}$ to $\widetilde{\mathcal{X}}$. By Proposition 3.2.5, such a deformation is unique up to an isomorphism. We thereby obtain an automorphism $h: \widetilde{X}_{0} \rightarrow \widetilde{X}_{0}$ such that the morphisms $\widetilde{P} \circ h$ and $g \circ \widetilde{P}$ are 2-isomorphic. Let $\tau: \widetilde{P} \circ h \Rightarrow g \circ \widetilde{P}$ be a 2-isomorphism. Since we have the 2-isomorphism

$$
\widetilde{P} \circ h \circ \widetilde{s} \stackrel{\tau * \mathrm{id}_{\tilde{s}}}{\Longrightarrow} g \circ \widetilde{P} \circ \widetilde{s} \stackrel{\mathrm{id}_{\tilde{s}} * \epsilon}{\Longrightarrow} g \circ \widetilde{P} \circ \widetilde{t} \stackrel{\tau^{-1}}{\Longrightarrow} \Longrightarrow
$$

then there is a morphism of algebraic spaces

$$
h \times h: \widetilde{X}_{1}=\widetilde{X}_{0} \times \widetilde{\mathcal{X}}_{0} \rightarrow \widetilde{X}_{0} \times_{\widetilde{\mathcal{X}}} \widetilde{X}_{0}=\widetilde{X}_{1} .
$$

It is easy to check that $(h, h \times h)$ is an automorphism of $\widetilde{\mathcal{G}}$. Since the diagram

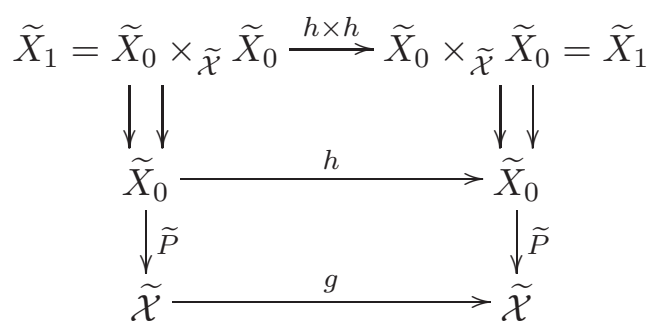

is 2-commutative, $C_{\widetilde{\mathcal{G}}}(h, h \times h)$ is a 2-isomorphism class of $g$, and therefore $C_{\widetilde{\mathcal{G}}}$ is surjective. 
We define a homomorphism $A: \operatorname{Aut}_{\widetilde{D e f m}_{\mathcal{X}}\left(X_{0}, \widetilde{\mathcal{X}}\right)}\left(\widetilde{X}_{0}\right) \rightarrow \operatorname{Aut}_{\operatorname{Defm}_{S}(\mathcal{G}, \widetilde{S})}(\widetilde{\mathcal{G}})$ by $A\left(h: \widetilde{X}_{0} \rightarrow \widetilde{X}_{0}\right)=$ $\mathrm{sq}_{2}\left(\operatorname{cosq}_{0}^{\widetilde{\mathcal{X}}}(h)\right)$. Since the diagram

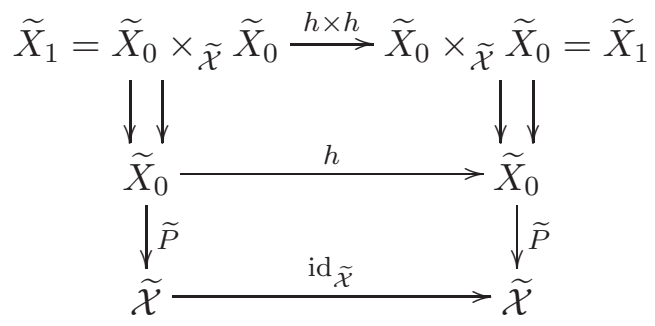

is 2-commutative, $C_{\widetilde{\mathcal{G}}} \circ A(h)$ is the 2-isomorphism class of $\operatorname{id}_{\tilde{\mathcal{X}}}$.

Conversely, let $\left(h_{0}, h_{1}\right): \widetilde{\mathcal{G}} \rightarrow \widetilde{\mathcal{G}}$ be an automorphism of $\operatorname{Defm}_{S}(\mathcal{G}, \widetilde{S})$ with $C_{\widetilde{\mathcal{G}}}\left(h_{0}, h_{1}\right)=\operatorname{id}_{\widetilde{\mathcal{X}}}$. Then $h_{0}$ is an automorphism of $\operatorname{Defm}_{\mathcal{X}}\left(X_{0}, \widetilde{\mathcal{X}}\right)$ and the isomorphism

$$
\operatorname{cosq}_{0}^{\tilde{\mathcal{X}}}: \operatorname{Aut}_{\widetilde{\mathcal{X}}}\left(\widetilde{X_{0}}\right) \stackrel{\sim}{\rightarrow} \operatorname{Aut}_{\widetilde{\mathcal{X}}}\left(\widetilde{X_{\bullet}}\right)
$$

induces $h_{\bullet}=\operatorname{cosq}_{0}^{\tilde{\mathcal{X}}}\left(h_{0}\right)$, and hence $\left(h_{0}, h_{1}\right)=A\left(h_{0}\right)$.

Since the category of 1 -morphisms from $\widetilde{\mathcal{X}}$ to $\widetilde{\mathcal{X}}$ is equivalent to that of morphisms from the groupoid space $\widetilde{\mathcal{G}}$ to $\widetilde{\mathcal{X}}$ (from [LM00, Lemma 3.2]), the 2-automorphism group of id $\tilde{\mathcal{X}}_{\text {in }} \operatorname{Defm}_{S}(\mathcal{X}, \widetilde{S})$ is isomorphic to the group of 2-automorphisms $\alpha$ of $\widetilde{P}$ in $\widetilde{\mathcal{X}}\left(\widetilde{X}_{0}\right)$, so that the induced 2-isomorphism $\widetilde{P} \widetilde{s} \Rightarrow \widetilde{P} \widetilde{t}$ is equal to $\epsilon$ and $\alpha \times \operatorname{id}_{S}: \widetilde{P} \times \operatorname{id}_{S} \Rightarrow \widetilde{P} \times \operatorname{id}_{S}$ is the identity. This is the group of automorphisms of $\widetilde{X}_{0}$ in $\operatorname{Defm}_{\mathcal{X}}\left(X_{0}, \widetilde{\mathcal{X}}\right)$ which induces the identity of $\operatorname{Defm}_{S}(\widetilde{\mathcal{G}}, \widetilde{S})$, i.e. the kernel of $A$.

\subsection{The Ext groups}

We refer to $[\mathrm{Ols} 02, \S 2.11]$ for the definition of the Ext groups of the cotangent complex.

Lemma 3.4.1. There is a natural isomorphism

$$
\operatorname{Ext}^{i}\left(L_{X_{\bullet} / \mathcal{X}}, P_{\bullet}^{*} J\right) \cong \operatorname{Ext}^{i}\left(\Omega_{X_{0} / \mathcal{X}}, P^{*} J\right) \quad(i \geqslant 0)
$$

and the right-hand side is zero for $i>0$.

Proof. Since $X_{\bullet}$ is smooth over $\mathcal{X}$, then $L_{X \bullet / \mathcal{X}}$ is quasiisomorphic to $\Omega_{X \bullet / \mathcal{X}}$. Then the isomorphism follows from [Ols02, Lemma 4.7(i)]. The right-hand side is zero for $i>0$ by Lemma 3.2.4.

Proposition 3.4.2. Let $J$ be a quasicoherent sheaf on $\mathcal{X}$.

1) There are natural isomorphisms

$$
\operatorname{Ext}^{i}\left(L_{\mathcal{X} / S}, J\right) \cong \operatorname{Ext}^{i}\left(L_{X_{\bullet} / S_{\bullet}}, P_{\bullet}^{*} J\right) \quad(i>0) .
$$

2) There is an exact sequence

$$
\begin{aligned}
0 \rightarrow & \operatorname{Ext}^{-1}\left(L_{\mathcal{X} / S}, J\right) \rightarrow \operatorname{Ext}^{0}\left(L_{X_{0} / \mathcal{X}}, P^{*} J\right) \\
& \stackrel{B}{\longrightarrow} \operatorname{Ext}^{0}\left(L_{X \bullet / S_{\bullet}}, P_{\bullet}^{*} J\right) \rightarrow \operatorname{Ext}^{0}\left(L_{\mathcal{X} / S}, J\right) \rightarrow 0 .
\end{aligned}
$$

Proof. Fix $n \geqslant 4$. Then for $i \leqslant 3$ we have isomorphisms

$$
\begin{aligned}
\operatorname{Ext}^{i}\left(L_{X \bullet / S}, P_{\bullet}^{*} J\right) & \cong \operatorname{Ext}^{i}\left(\tau_{\geqslant-n} L_{X \bullet / S}, P_{\bullet}^{*} J\right), \\
\operatorname{Ext}^{i}\left(L_{X_{\bullet} / \mathcal{X}}, P_{\bullet}^{*} J\right) & \cong \operatorname{Ext}^{i}\left(L_{X_{\bullet} / \mathcal{X}}^{\geqslant-n}, P_{\bullet}^{*} J\right), \\
\operatorname{Ext}^{i}\left(L_{\mathcal{X} / S}, J\right) & \cong \operatorname{Ext}^{i}\left(L_{\mathcal{X} / S}^{\geqslant-n}, J\right) .
\end{aligned}
$$




\section{DEFORMATION THEORY OF ALGEBRAIC STACKS}

By the equivalence of triangulated categories

$$
P_{\bullet}^{*}: D_{\mathrm{qcoh}}^{b}\left(O_{\mathcal{X}}\right) \stackrel{\sim}{\longrightarrow} D_{\mathrm{qcoh}}^{b}\left(O_{X_{\bullet}}\right)
$$

as in [LM00, Proposition 13.2.4], we deduce the isomorphisms

$$
\operatorname{Ext}^{i}\left(L_{\mathcal{X} / S}^{\geqslant-n}, J\right) \cong \operatorname{Ext}^{i}\left(L P_{\bullet}^{*} L_{\mathcal{X} / S}^{\geqslant-n}, P_{\bullet}^{*} J\right) .
$$

The morphisms

$$
X \bullet \stackrel{P_{\bullet}}{\longrightarrow} \mathcal{X} \stackrel{x}{\longrightarrow} S
$$

induce a triangle in $D^{+}\left(O_{X_{\bullet}}\right)$ :

$$
L P_{\bullet}^{*} L_{\mathcal{X} / S}^{\geqslant-n} \rightarrow L_{X_{\bullet} / S}^{\geqslant-n} \rightarrow M \rightarrow L P_{\bullet}^{*} L_{\mathcal{X} / S}^{\geqslant-n}[1] .
$$

Here $M$ is an object in $D^{+}\left(O_{X_{\bullet}}\right)$ and there is an isomorphism $\tau_{\geqslant-n} L_{X \bullet / \mathcal{X}} \stackrel{\sim}{\rightarrow} \tau_{\geqslant-n} M$ (from [LM00, Theorem-Definition 17.3(3) and Remark 17.4(3)]).

The desired results follows from the long exact sequence induced by this triangle, the isomorphisms (3.4.2.1)-(3.4.2.4) and Lemma 3.4.1.

The following proposition concludes the proof of Theorem 1.2.

Proposition 3.4.3. The diagram

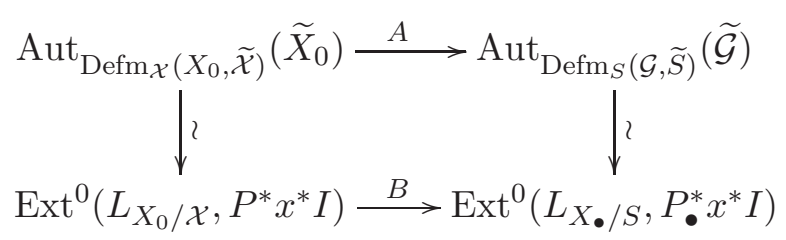

is commutative. Here the horizontal arrows are those in Propositions 3.3.2 and 3.4.2 and the vertical isomorphisms are those in [Ols02, Theorem 1.4] and [Ill71, ch. III, Theorem 2.1.7].

Proof. Applying the cosquelette functor, we have a commutative diagram of isomorphisms.

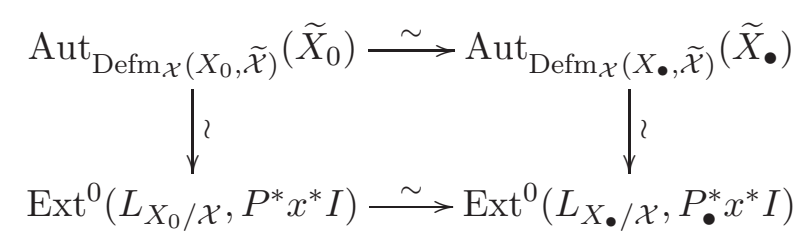

The proposition follows from the fact that the isomorphism in [Ols02, Theorem 1.4(3)] is functorial by arguments similar to those in [Ols02, Remark 2.24].

\section{Versal deformation}

\subsection{Definitions and preliminary results}

Let $k$ be a field. Let $\left(\operatorname{Art}_{k}\right)$ be the category of artinian local $k$-algebras whose residue field is isomorphic to $k$. Morphisms of $\left(\mathrm{Art}_{k}\right)$ are local homomorphisms.

Let $\mathcal{X}$ be an algebraic stack over $\operatorname{Spec} k$.

Definition 4.1.1. Let $R$ be a complete local Noetherian ring with residue field $k$ and $\mathcal{Y}$ a deformation of $\mathcal{X}$ to $\operatorname{Spec} R$. The pair $(R, \mathcal{Y})$ is said to be a versal deformation of $\mathcal{X}$ if it satisfies the following conditions:

1) If $A \in\left(\operatorname{Art}_{k}\right)$ and $\widetilde{\mathcal{X}}$ is a deformation of $\mathcal{X}$ to $\operatorname{Spec} A$, then there exists a local homomorphism $f: R \rightarrow A$ such that $\widetilde{\mathcal{X}}$ is 1-isomorphic to $\widetilde{\mathcal{Y}} \times \operatorname{Spec} R \operatorname{Spec} A$.

2) If $A=k[\epsilon] /\left(\epsilon^{2}\right)$, then such an $f$ is unique. 


\section{AOKI}

We define the functor $D_{\mathcal{X}}:\left(\operatorname{Art}_{k}\right) \rightarrow($ Sets) as follows:

$$
D_{\mathcal{X}}(A)=\text { the set of isomorphism classes of flat deformations of } \mathcal{X} \text { to } \operatorname{Spec} A \text {. }
$$

The existence of a versal deformation of $\mathcal{X}$ is equivalent to the existence of a prorepresentable hull of the functor $D_{\mathcal{X}}$, in the sense of [Sch68, Definition 2.7]. We have criterion for the existence of a prorepresentable hull.

Theorem 4.1.2 [Sch68, Theorem 2.11]. Let $A^{\prime} \rightarrow A$ and $A^{\prime \prime} \rightarrow A$ be morphisms in $\left(\right.$ Art $\left._{k}\right)$. Consider the natural map

$$
D_{\mathcal{X}}\left(A^{\prime} \times_{A} A^{\prime \prime}\right) \rightarrow D_{\mathcal{X}}\left(A^{\prime}\right) \times_{D_{\mathcal{X}}(A)} D_{\mathcal{X}}\left(A^{\prime \prime}\right) .
$$

Then $D_{\mathcal{X}}$ has a prorepresentable hull if and only if the following conditions are satisfied:

$\mathrm{H1}$ ) if $A^{\prime \prime} \rightarrow A$ is a small extension, then (4.1.2.1) is a surjection;

H2) the map (4.1.2.1) is a bijection when $A=k, A^{\prime \prime}=k[\epsilon]$;

H3) $\operatorname{dim}\left(D_{\mathcal{X}}\left(k[\epsilon] /\left(\epsilon^{2}\right)\right)\right)<\infty$.

\subsection{Existence of a prorepresentable hull}

Lemma 4.2.1. If we replace $\mathcal{X}$ by a simplicial algebraic space $X_{\bullet}$, then the condition $H 1$ in Theorem 4.1.2 holds.

Proof. Let $Y_{\bullet}$ (respectively $Y_{\bullet}^{\prime}$, respectively $Y_{\bullet}^{\prime \prime}$ ) be a flat deformation of $X_{\bullet}$ to $\operatorname{Spec} A$ (respectively $\operatorname{Spec} A^{\prime}$, respectively $\left.\operatorname{Spec} A^{\prime \prime}\right)$ and suppose that $Y_{\bullet}^{\prime}$ and $Y_{\bullet}^{\prime \prime}$ restricted on $\operatorname{Spec} A$ are isomorphic to $Y_{\bullet}$. We show that there exists a flat deformation $Z_{\bullet}$ of $X_{\bullet}$ to $\operatorname{Spec} A^{\prime} \times_{A} A^{\prime \prime}$ whose restrictions on $\operatorname{Spec} A^{\prime}$ and $\operatorname{Spec} A^{\prime \prime}$ are isomorphic to $Y_{\bullet}^{\prime}$ and $Y_{\bullet}^{\prime \prime}$.

Let $O_{Z_{\bullet}}$ be the ring object $O_{Y_{\bullet}^{\prime}} \times_{O_{Y_{\bullet}}} O_{Y_{\bullet}^{\prime \prime}}$ in the topos of étale sheaves on $X_{\bullet}$. Since deformations of $X_{\bullet}$ are equivalent to those of the ringed topos $\left(\left|X_{\bullet}\right|, O_{X_{\bullet}}\right)$ of étale $O_{X_{\bullet}}$-modules, $O_{Z_{\bullet}}$ gives the required simplicial algebraic space.

Proposition 4.2.2. The condition H1 in Theorem 4.1.2 holds.

Proof. Let $\mathcal{Y}$ (respectively $\mathcal{Y}^{\prime}$, respectively $\mathcal{Y}^{\prime \prime}$ ) be a flat deformation of $\mathcal{X}$ to $\operatorname{Spec} A$ (respectively Spec $A^{\prime}$, respectively $\operatorname{Spec} A^{\prime \prime}$ ) and suppose that $\mathcal{Y}^{\prime}$ and $\mathcal{Y}^{\prime \prime}$ restricted on $\operatorname{Spec} A$ are isomorphic to $\mathcal{Y}$. We show that there exists a flat deformation $\mathcal{Z}$ of $\mathcal{X}$ to $\operatorname{Spec} A^{\prime} \times_{A} A^{\prime \prime}$ whose restrictions on $\operatorname{Spec} A^{\prime}$ and $\operatorname{Spec} A^{\prime \prime}$ are isomorphic to $\mathcal{Y}^{\prime}$ and $\mathcal{Y}^{\prime \prime}$.

Let $P: X_{0} \rightarrow \mathcal{X}$ be a presentation and $X_{\bullet}=\operatorname{cosq}_{0}^{\mathcal{X}}\left(X_{0}\right)$ the associated simplicial algebraic space. We may suppose that $X_{0}$ is an affine scheme or a disjoint union of affine schemes.

By [Ols02, Theorem 1.4], Lemmas 3.2.4 and Lemma 3.2.1, there exists a flat deformation $Y_{0}$ (respectively $Y_{0}^{\prime}$, respectively $Y_{0}^{\prime \prime}$ ) of $X_{0}$ to $\operatorname{Spec} A$ (respectively $\operatorname{Spec} A^{\prime}$, respectively $\operatorname{Spec} A^{\prime \prime}$ ) which gives a presentation $Y_{0} \rightarrow \mathcal{Y}$ (respectively $Y_{0}^{\prime} \rightarrow \mathcal{Y}^{\prime}$, respectively $Y_{0}^{\prime \prime} \rightarrow \mathcal{Y}^{\prime \prime}$ ). By Lemma 3.2.3, the associated simplicial algebraic space $Y_{\bullet}=\operatorname{cosq}_{0}^{\mathcal{Y}}(Y)$ (respectively $Y_{\bullet}^{\prime}=\operatorname{cosq}_{0}^{\mathcal{Y}^{\prime}}\left(Y_{0}^{\prime}\right)$, respectively $\left.Y_{\bullet}^{\prime \prime}=\operatorname{cosq}_{0}^{\mathcal{Y}^{\prime \prime}}\left(Y_{0}^{\prime \prime}\right)\right)$ is a flat deformation of $X_{\bullet}$ to $\operatorname{Spec} A$ (respectively $\operatorname{Spec} A^{\prime}$, respectively $\operatorname{Spec} A^{\prime \prime}$ ) 


\section{DEFORMATION THEORY OF ALGEBRAIC STACKS}

and the restrictions of $Y_{\bullet}^{\prime}$ and $Y_{\bullet}^{\prime \prime}$ on $\operatorname{Spec} A$ are isomorphic to $Y_{\bullet}$.

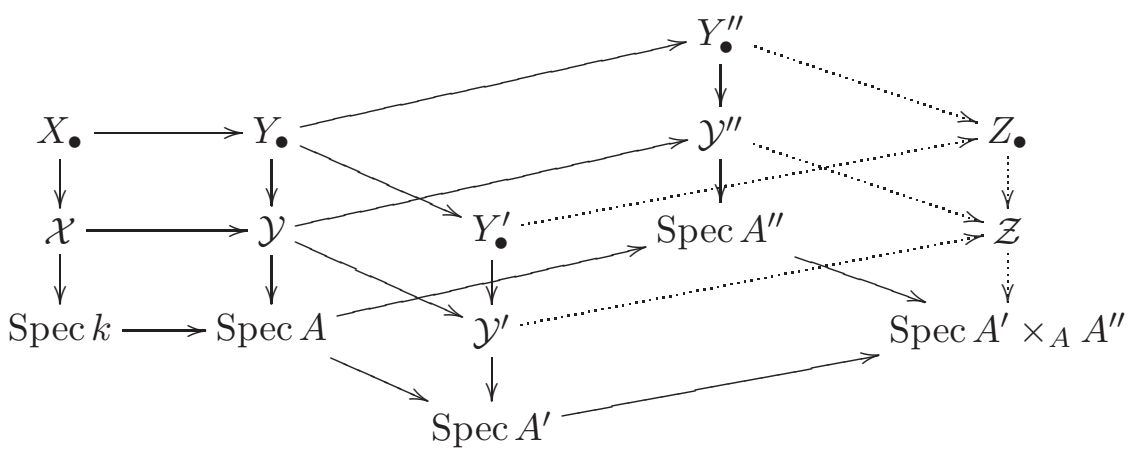

By Lemma 4.2.1, there exists a simplicial algebraic space $Z_{\bullet}$ which is a deformation of $X_{\bullet}$ to $\operatorname{Spec} A^{\prime} \times_{A} A^{\prime \prime}$ and whose restrictions on $\operatorname{Spec} A^{\prime}$ and $\operatorname{Spec} A^{\prime \prime}$ are isomorphic to $Y_{\bullet}^{\prime}$ and $Y_{\bullet}^{\prime \prime}$. By Proposition 3.1.3 and Corollary 3.1.5, there exists an algebraic stack $\mathcal{Z}$, as required.

Proposition 4.2.3. The condition H2 in Theorem 4.1.2 holds.

Proof. The argument of [Sch68, p. 220] for schemes also applies to our case.

The following proposition completes the proof of Theorem 1.3.

Proposition 4.2.4. If $\mathcal{X}$ is proper over $k$, then the functor $D \mathcal{X}$ satisfies the condition $H 3$ of Theorem 4.1.2.

Proof. By Theorems 1.2 and 4.1.2, it is enough to show that the $k$-vector space $\operatorname{Ext}^{1}\left(L_{\mathcal{X} / k}, O_{\mathcal{X}}\right)$ is finite-dimensional.

Let $x: \mathcal{X} \rightarrow$ Spec $k$ be the structural morphism. Then the isomorphism of derived functors

$$
R x_{*} R \mathcal{H o m}\left(-, O_{\mathcal{X}}\right) \cong R \operatorname{Hom}\left(-, O_{\mathcal{X}}\right): D\left(O_{\mathcal{X}}\right)^{\text {op }} \rightarrow D(k)
$$

induces the spectral sequence

$$
E_{2}^{p q}=R^{p} x_{*} \mathcal{E} x t^{q}\left(L_{\mathcal{X} / k}, O_{\mathcal{X}}\right) \Rightarrow \operatorname{Ext}^{p+q}\left(L_{\mathcal{X} / k}, O_{\mathcal{X}}\right) .
$$

Therefore it suffices to show that $R^{p} x_{*} \mathcal{E} x t^{q}\left(L_{\mathcal{X} / k}, O_{\mathcal{X}}\right)$ is finite-dimensional for all $p$ and $q$.

Let $X_{0} \rightarrow \mathcal{X}$ be a presentation and let $X_{\bullet}=\operatorname{cosq}_{0}^{\mathcal{X}}\left(X_{0}\right)$. Since $L_{X_{\bullet} / k}$ is a coherent $O_{X_{\bullet}}$-module, $L_{\mathcal{X} / k}$ is also coherent. Therefore $R \mathcal{H} \operatorname{Hom}\left(L_{\mathcal{X} / k}, O_{\mathcal{X}}\right) \in D_{\text {coh }}\left(O_{\mathcal{X}}\right)$ by [LM00, Theorem 15.6] and $\mathcal{E}_{x} t^{q}\left(L_{\mathcal{X} / k}, O_{\mathcal{X}}\right)$ is coherent for all $q$. By [Fal03, Theorem 1], $R^{p} x_{*} \mathcal{E} x t^{q}\left(L_{\mathcal{X} / k}, O_{\mathcal{X}}\right)$ is a coherent $k$-module for all $p$ and $q$, i.e. it is a finite-dimensional $k$-vector space.

\section{Example}

Let $g: G \rightarrow S$ be a group scheme over a scheme $S$. The classifying stack $B G$ is defined by

$$
B G(U)=\text { the category of principle } G \text {-bundles over } U \text {. }
$$

If $G$ is smooth, separated and of finite presentation, then $B G$ is an algebraic stack and the natural morphism $P: S \rightarrow B G$ corresponding to the trivial $G$-bundle over $S$ gives a presentation [LM00, Example 4.6.1]. Moreover, the associated simplicial algebraic space $G_{\bullet}$ is the nerve of $G$ [Ill71, ch. VI, § 2.1, 9.3.1].

Suppose that $S$ is an affine scheme. By Proposition 3.2.5, the set of 1-isomorphism classes of deformations of $B G$ is isomorphic to that of $G_{\bullet}$, which is the set of isomorphism classes of deformations of $G$ as a group scheme [Ill71, ch. IV, $\S$ 9.3.1 and ch. V, Remark 1.2.4.1]. 


\section{DEFORMATION THEORY OF ALGEBRAIC STACKS}

Let $B \widetilde{G}$ be a deformation of $B G$ to $\widetilde{S}$. Consider the exact sequence in Proposition 3.3.2:

$$
\begin{aligned}
0 \rightarrow & 2-\operatorname{Aut}_{\operatorname{Defm}_{S}^{2}(B G, \widetilde{S})}\left(\operatorname{id}_{B \widetilde{G}}\right) \rightarrow \operatorname{Aut}_{\operatorname{Defm}_{B G}(S, B \widetilde{G})}(\widetilde{S}) \\
& \stackrel{A}{\longrightarrow} \operatorname{Aut}_{\operatorname{Defm}_{S}\left(G_{\bullet}, \widetilde{S}\right)}(\widetilde{G} \bullet) \rightarrow \operatorname{Aut}_{\operatorname{Defm}_{S}^{1}(B G, \widetilde{S})}(B \widetilde{G}) \rightarrow 0 .
\end{aligned}
$$

The group $\operatorname{Aut}_{\operatorname{Defm}_{S}\left(G_{\bullet}, \widetilde{S}\right)}\left(\widetilde{G}_{\bullet}\right)$ is the automorphism group of $\widetilde{G}$ as a deformation of a group scheme.

The group $\operatorname{Aut}_{\operatorname{Defm}_{B G}(S, B \widetilde{G})}(\widetilde{S})$ is the group of automorphisms $\tau: \widetilde{S} \rightarrow \widetilde{S}$ and 2-isomorphisms $\widetilde{P} \circ \tau \Rightarrow \widetilde{P}$. Since $B \widetilde{G}$ is a stack over $\widetilde{S}$, then $\tau$ must be the identity. Therefore $\operatorname{Aut}_{\operatorname{Defm}_{B G}(S, B \widetilde{G})}(\widetilde{S})$ is the group of automorphisms of $\widetilde{P}$ in the category $B \widetilde{G}(\widetilde{S})$ whose restriction on $P$ is the identity. This is the automorphism group of $\widetilde{G}$ as a deformation of a group scheme, and the homomorphism $A$ is an isomorphism.

Hence the groups 2-Aut $\operatorname{Defm}_{S}^{2}(B G, \widetilde{S})\left(\mathrm{id}_{B \widetilde{G}}\right)$ and $\operatorname{Aut}_{\operatorname{Defm}_{S}^{1}(B G, \widetilde{S})}(B \widetilde{G})$ are zero.

\section{ACKNOWLEDGEMENTS}

The author would like to express his thanks to Professor F. Kato and Dr. M. Olsson for valuable suggestions and advice on this paper, and to Professor G. Laumon for introducing the paper [Fal03]. The author is grateful to Professor A. Moriwaki for his encouragement.

The author also thanks the referee for helpful comments, which led to simplifications of a number of arguments.

\section{REFERENCES}

DiG61 J. Dieudonné and A. Grothendieck, Éléments de géométrie algébrique, Publ. Math. Inst. Hautes Études Sci. 4, 8, 11, 17, 20, 24, 28, 32 (1961-1967).

Bou72 N. Bourbaki, Commutative algebra, elements of mathematics (Hermann, Paris, 1972).

Del74 P. Deligne, Théorie de Hodge III, Publ. Math. Inst. Hautes Études Sci. 44 (1974), 5-78.

DM69 P. Deligne and D. Mumford, The irreducibility of moduli space of curves of given genus, Publ. Math. Inst. Hautes Études Sci. 36 (1969), 75-109.

Fal03 G. Faltings, Finiteness of coherent cohomology for proper fppf stacks, J. Algebraic Geom. 12 (2003), $357-366$.

Gir71 J. Giraud, Cohomologie non abélienne, Grundlehren Math. Wiss., vol. 179 (Springer, Berlin, 1971).

Ill71 L. Illusie, Complexe cotangent et déformations I, II, Lecture Notes in Mathematics, vols 239, 283 (Springer, Berlin, 1971).

Knu71 D. Knutson, Algebraic spaces, Lecture Notes in Mathematics, vol. 203 (Springer, Berlin, 1971).

LM00 G. Laumon and L. Moret-Bailly, Champs algébriques, Ergeb. Math. Grenzgeb. (3), vol. 39 (Springer, Berlin, 2000).

Ols02 M. Olsson, Deformation theory of 1-morphisms to algebraic stacks, Preprint (2002), www-math.mit.edu/ molsson/1def.ps

Sch68 M. Schlessinger, Functors of Artin rings, Trans. Amer. Math. Soc. 130 (1968), 208-222.

SGA3 M. Demazure and A. Grothendieck, Schémas en groupes, Lecture Notes in Mathematics, vols 151, 152, 153 (Springer, Berlin, 1962-1970).

SGA4 M. Artin, A. Grothendieck and J. L. Verdier, Théorie de topos et cohomologie étale des schémas, Lecture Notes in Mathematics, vols 269, 270, 305 (Springer, Berlin, 1971).

SGA7 A. Grothendieck, P. Deligne and N. Katz, Groupes de monodromie en géométrie algébrique, Lecture Notes in Mathematics, vols 288, 340 (Springer, Berlin, 1972-1973).

Masao Aoki aoki@math.kyoto-u.ac.jp

Department of Mathematics, Kyoto University, Sakyo-ku, Kyoto 606-8502, Japan 